\title{
Alguns aspectos conceituais e práticos do método de Mohr na determinação de cloridrato de metformina em formulações farmacêuticas
}

\author{
Some conceptual aspects and practice of Mohr method in the \\ determination metformin hydrochloride in pharmaceutical formulations
}

\author{
Willian Toito Suarez'; Elen Romão Sartori²; Orlando Fatibello-Filho ${ }^{3}$
}

\begin{abstract}
Resumo
Neste trabalho, aspectos conceituais e práticos do método de Mohr na determinação de cloridrato de metformina em formulações farmacêuticas são descritos. Este experimento é simples e pode ser uma boa alternativa para ser aplicado em uma aula experimental em um curso de gradução.

Palavras-chave: Cloridrato de metformina. Método de Mohr. Argentimetria. Determinação de cloreto.
\end{abstract}

\begin{abstract}
In this paper conceptual aspects and practices of Mohr method in the determination of metformin hydrochloride in pharmaceutical formulations are described. This experiment is simple and might be a good alternative to be applied in an experimental subject in undergraduation courses.
\end{abstract}

Keywords: Metformin hydrochloride. Mohr method. Argentimetry. Chloride determination.

\section{Introdução}

Os aspectos do cotidiano e a interdisciplinaridade têm sido incentivados pelas Leis de Diretrizes e Bases da Educação Nacional (BRASIL, 1996) como forma de desencadear ações que busquem reestruturar as bases curriculares e metodológicas em todos os níveis educacionais, principalmente no modelo de curso superior, no intuito de aproximar as lições de química abordadas na sala de aula a algo presente no cotidiano dos estudantes e de alguma forma, induzindo esses a relacionarem as ciências Químicas com o contexto cultural, socioeconômico e político, acompanhando e compreendendo os avanços científico-tecnológicos e educacionais. A contextualização de experimentos atrai a atenção dos estudantes para o conteúdo abordado e induz o conhecimento à visão crítica, instiga à capacidade de relacionar fatos, reconhecer a linguagem química e revelar certas concepções pré-existentes sobre os conceitos químicos, despertando de alguma forma o interesse científico em alunos que poderão vir a ser futuros pesquisadores.

Uma aprendizagem efetiva ocorre quando o estudante consegue realizar conexões entre o conteúdo que está sendo introduzido pelo professor e seu conhecimento prévio. Dessa forma, as

\footnotetext{
${ }^{1}$ Docente do Departamento de Química da Universidade Federal de Viçosa: williants@hotmail.com.

${ }^{2}$ Docente do Departamento de Química da Universidade Estadual de Londrina: elensartori@uel.br.

${ }^{3}$ Professor Titular da Universidade Federal de São Carlos: bello@ufscar.br.
} 
situações novas, em que se propõem experimentos que relacionem o cotidiano ao conteúdo ministrado pelo professor, fazem com que os alunos exercitem o lado crítico e científico, promovendo um maior envolvimento no processo ensino-aprendizagem, trazendo a tona o conhecimento prévio $\mathrm{e}$ relacionando-os ao experimento realizado, e além de tudo, incentivando a capacidade dos mesmos em organizar e gerir o seu aprendizado, importantes para a formação de profissionais da área de química. Sempre pensando na melhoria do ensino de Química, alguns trabalhos de contextualização de experimentos têm sido amplamente explorados neste grupo de trabalho (FATIBELLO-FILHO et al., 2006; LUPETTI et al., 2005; RAMOS et al. 2000; SARTORI; BATISTA; FATIBELLOFILHO, 2008; SARTORI et al. 2009; SUAREZ; FERREIRA; FATIBELLO-FILHO, 2007; VIEIRA; FIGUEIREDO-FILHO; FATIBELLO-FILHO, 2007), principalmente empregando materiais alternativos simples e facilmente disponíveis no cotidiano dos estudantes.

Dentre as técnicas volumétricas clássicas, o método de Mohr destaca-se na determinação de cloreto (JEFFERY etal., 1989), por meio da formação de um precipitado colorido, e de certa forma, quase sempre restrito a determinação desse ânion em água potável, amostras de água do mar ou ainda, em soluções de $\mathrm{NaCl}$ previamente preparadas para fins didáticos. Por empregar solução de nitrato de prata, é também chamado como de método argentimétrico. Gradualmente, o seu emprego foi sendo reduzido, devido ao desenvolvimento de outras técnicas analíticas mais rápidas e com menores limites de detecção (HARRIS, 2005). Porém, esta técnica ainda representa uma ferramenta útil para auxiliar no aprendizado de química, mais especificamente, na compreensão de equilíbrio químico.

Basicamente, na análise titulométrica ou volumétrica (HARRIS, 2005; OHLWEILER, 1980) é medido o volume de reagente (titulante) necessário para ocorrer uma reação estequiométrica com o analito a ser determinado, cuja mudança de cor do indicador, por exemplo, indica o ponto final da reação. Já o ponto de equivalência é o ponto da titulação em que a quantidade da solução do titulante adicionada é equivalente à substância com o qual irá reagir (analito). O ponto de equivalência e o ponto final não coincidem necessariamente. A diferença entre os mesmos (ponto estequiométrico) é o erro da titulação, que se reduz subtraindo-se a titulação do branco dos resultados. A determinação realizada dessa forma é denominada quantitativa, estabelecendo uma quantidade exata da substância a ser analisada.

No método de Mohr, uma solução contendo íons cloreto é titulada com solução de nitrato de prata $\left(\mathrm{AgNO}_{3}\right)$ na presença de uma pequena quantidade de indicador cromato de potássio $\left(\mathrm{K}_{2} \mathrm{CrO}_{4}\right)$, de cor amarela. $\mathrm{O}$ ponto final da titulação é identificado por uma mudança de coloração da solução, resultado da reação entre os íons cromato com os íons prata para formar o precipitado de cromato de prata $\left(\mathrm{Ag}_{2} \mathrm{CrO}_{4}\right)$, de cor vermelha e pouco solúvel (JEFFERY et al., 1989).

Existem fatores importantes a serem considerados no método de Mohr, a saber: o $\mathrm{pH}$ da solução e a concentração do indicador. A titulação deve ser conduzida em meio neutro ou levemente básico, em pH entre 6,5 e 9. Em soluções ácidas o cromato reage com os íons hidrogênio formando o hidrogenocromato, $\left(\mathrm{HCrO}_{4}^{-}\right)$e dicromato $\left(\mathrm{Cr}_{2} \mathrm{O}_{7}^{2-}\right)$ (Eq. 1) (JEFFERY et al., 1989) ocasionando a diminuição da concentração do $\mathrm{CrO}_{4}^{2-}$ e, consequentemente, o indicador deixa de funcionar ou há um erro considerável na determinação do ponto de equivalência:

$2 \mathrm{CrO}_{4}^{2-}+2 \mathrm{H}^{+} \leftrightarrows 2 \mathrm{HCrO}_{4}^{-} \leftrightarrows \mathrm{Cr}_{2} \mathrm{O}_{7}^{2-}+\mathrm{H}_{2} \mathrm{O}$

Por outro lado, em um pH superior a 9, ocorrerá a precipitação do hidróxido de prata $\left(\mathrm{K}_{\mathrm{s}}=2,3 \times\right.$ $10^{-8}$ ) (BACCAN et al., 2001), que posteriormente se decompõe em $\mathrm{Ag}_{2} \mathrm{O}$ e água (Eq. 2),

$2 \mathrm{Ag}^{+}+2 \mathrm{OH}^{-} \leftrightarrows 2 \mathrm{AgOH} \leftrightarrows \mathrm{Ag}_{2} \mathrm{O}+\mathrm{H}_{2} \mathrm{O}$ (Eq. 2) 
No que se diz respeito à concentração do indicador, recomenda-se usar uma solução de cromato de potássio mais diluída que a solução de cloreto a ser titulada, uma vez que em elevada concentração dá à solução a ser titulada uma cor vermelha muito forte, tornando difícil observar o início da precipitação do cromato de prata (JEFFERY et al., 1989).

O cloridrato de metformina (MET) é uma droga com propriedades antiiperglicêmicas, que normaliza os níveis elevados de glicose no sangue, consistindo um dos medicamentos de escolha inicial para o tratamento do diabetes tipo 2 (SILVA, 2002). Sua estrutura química é mostrada na Figura 1. A análise química desse medicamento é realizada cotidianamente em indústrias farmacêuticas ou em farmácias de manipulação para a verificação de seu princípio ativo, se está em concentração apropriada ao consumo humano.

Figura 1 - Fórmula estrutural do cloridrato de metformina.<smiles>CN(C)C(=N)NC(=N)N</smiles>

Fonte: Dados do autor.

Visando uma modificação nos experimentos tradicionais que são aplicados nas disciplinas de Química Analítica para estudantes de graduação de química ou de farmácia, nesse trabalho é apresentada uma contextualização dos princípios fundamentais envolvidos no método de Mohr para a determinação do teor de cloridrato de metformina em formulações farmacêuticas.

\section{Experimental}

\section{Reagentes e soluções}

Todas as soluções foram preparadas com água destilada e deionizada obtida de um sistema de purificação Milli-Q (Millipore, Bedford, MA), modelo UV Plus ultrabaixo teor de substâncias orgânicas dissolvidas. Todos os reagentes empregados foram de grau analítico e utilizados sem purificação prévia.

O cloridrato de metformina padrão foi adquirido da Sigma-Aldrich. As amostras dos medicamentos foram obtidas em drogarias locais. A solução estoque de MET 0,10 mol L-1 foi preparada dissolvendo-se $1,6600 \mathrm{~g}$ do fármaco em $100 \mathrm{~mL}$ de água destilada e deionizada.

A solução de $\mathrm{AgNO}_{3}$ (Sigma-Aldrich) 0,100 mol $\mathrm{L}^{-1}$ foi obtida pesando-se $8,5000 \mathrm{~g}$ desse sal seco e dissolvendo-se em $500 \mathrm{~mL}$ de água destilada e deionizada. A solução do indicador cromato de potássio $5 \% \mathrm{~m} / \mathrm{v}$ foi preparada pesando-se 2,5000 $\mathrm{g}$ de $\mathrm{K}_{2} \mathrm{CrO}_{4}$ (Merck) e dissolvendo-se em $50 \mathrm{~mL}$ de água destilada e deionizada.

\section{Instrumentação}

As titulações foram realizadas usando um Erlenmeyer de $500 \mathrm{~mL}$, uma bureta de $50 \mathrm{~mL}$ fixada em um suporte apropriado e uma pipeta volumétrica de $25 \mathrm{~mL}$.

Para a comparação dos resultados, as amostras foram analisadas usando um espectrofotômetro com arranjo linear de diodos Hewlett Packard, modelo 8452A, empregando-se cubeta de quartzo de $1,0 \mathrm{~cm}$ de caminho ótico acoplado a um microcomputador. As medidas de absorbância da solução de referência e dos medicamentos foram realizadas em água com detecção em $232 \mathrm{~nm}$.

\section{Preparo das amostras}

Duas amostras de medicamentos contendo cloridrato de metformina, denominadas A e B, de diferentes laboratórios, foram utilizadas. Essas continham uma concentração rotulada de 500 e 850 $\mathrm{mg}$ de cloridrato de metformina por comprimido, respectivamente. Para a preparação das amostras, cinco comprimidos de cada uma das amostras foram pesados em balança analítica e reduzidos a pó em um almofariz e pistilo. Considerando-se a 
dosagem de cloridrato de metformina declarada no rótulo de cada produto, uma quantidade adequada do comprimido foi dissolvida em água deionizada, sendo o volume completado com água em balão volumétrico de $100 \mathrm{~mL}$. Os excipientes insolúveis contidos nas amostras foram removidos por filtração simples em papel de filtro.

\section{Procedimento}

Alíquotas de $25 \mathrm{~mL}$ de soluções de referência ou da amostra contendo cloridrato de metformina em concentração aproximada de $0,1 \mathrm{~mol} \mathrm{~L}^{-1}$ foram transferidas para um Erlenmeyer de $500 \mathrm{~mL}$ com ajuda de uma pipeta volumétrica e adicionado $1 \mathrm{~mL}$ do indicador $\mathrm{K}_{2} \mathrm{CrO}_{4} 5 \% \mathrm{~m} / \mathrm{v}$. Em seguida, foram tituladas com solução de $\mathrm{AgNO}_{3} \quad 0,10$ mol L $\mathrm{L}^{-1}$, proveniente de uma bureta de $25 \mathrm{~mL}$, até o aparecimento de uma coloração vermelha (KOLTHOFF et al., 1969). Anotou-se o volume da solução de $\mathrm{AgNO}_{3}$ gasto e então com base nos dados obtidos em cada titulação e nos valores de massa dos comprimidos pesados, calculou-se a concentração de metformina por comprimido. Cada amostra foi titulada em triplicata.

A titulação envolve a adição da solução titulante, $\mathrm{AgNO}_{3}$, em pequenos incrementos até a observação do ponto final. É recomendável que a ponta da bureta não deve ficar posicionada muito acima do Erlenmeyer, para evitar projeções com consequente perda de reagente quando as gotas atingirem a solução de metformina (OHLWEILER, 1980). O Erlenmeyer deve ser colocado sobre um fundo branco, como por exemplo, uma folha de papel, para facilitar a visualização da mudança de coloração no ponto final da titulação.

Caso haja alguma dúvida se o ponto final foi ou não atingido, faz-se a leitura do volume da bureta e adiciona-se mais uma gota e observa-se o resultado. Os valores de volume de equivalência obtidos de cada amostra foram utilizados para calcular a concentração de metformina em mg/ comprimido. Os resultados foram comparados com o valor indicado no rótulo do medicamento e com os valores obtidos empregando-se um método espectrofotométrico da Farmacopéia Britânica (METFORMIN HYDROCHLORIDE, 1998).

Como branco analítico foi utilizado $25 \mathrm{~mL}$ de água destilada e deionizada contendo $1,0 \mathrm{~mL}$ do indicador $\mathrm{K}_{2} \mathrm{CrO}_{4}$ que, na ausência do fármaco, é titulado diretamente com $\mathrm{AgNO}_{3}$. Esse valor deve ser subtraído do volume de solução de nitrato de prata gasto na titulação do cloridrato de metformina, tanto do padrão quanto das amostras, como forma de corrigir o erro cometido na detecção do ponto final (BACCAN et al., 2001).

\section{Resultados e Discussão}

Inicialmente, mediu-se o valor do $\mathrm{pH}$ da solução aquosa de cloridrato de metformina $0,10 \mathrm{~mol} \mathrm{~L}^{-1}$ a ser titulada com solução de $\mathrm{AgNO}_{3}$. Esta medida é importante no sentido de verificar se o $\mathrm{pH}$ da solução está entre 6,5 e 9,0, para assegurar que o cloreto seja quantitativamente precipitado como cloreto de prata, antes que a precipitação do cromato de prata, de cor vermelho, seja perceptível. O pH da solução foi 7,1 , dentro do intervalo de $\mathrm{pH}$ indicado.

Foram realizadas três titulações sucessivas de $25,0 \mathrm{~mL}$ de solução padrão de cloridrato de metformina $0,10 \mathrm{~mol} \mathrm{~L}^{-1}$ com $\mathrm{AgNO}_{3} 0,10 \mathrm{~mol}$ $\mathrm{L}^{-1}$. Os volumes de nitrato de prata obtidos em cada uma dessas titulações $(24,8 ; 25,0$ e $25,1 \mathrm{~mL})$ apresentaram boa concordância quando comparado ao volume teórico de $25,0 \mathrm{~mL}$. Nessa titulação é o cloreto $\left(\mathrm{Cl}^{-}\right)$do cloridrato $(\mathrm{HCl})$ da molécula de metformina que reage com os íons $\mathrm{Ag}_{(\text {aq) }}^{+}$para formar o precipitado de cloreto de prata $\left(\mathrm{AgCl}_{(\mathrm{s})}\right)$. Como a relação estequiométrica entre $\mathrm{o}^{-}{ }_{(\mathrm{aq})}$ do cloridrato e a metformina é 1:1 (Figura 1), a seguinte equação foi empregada (Eq. 3):

$$
\mathrm{C}_{\mathrm{AgNO} 3} \times \mathrm{V}_{\mathrm{AgNO} 3}=\mathrm{C}_{\text {met }} \times \mathrm{V}_{\text {met }}
$$


sendo que $\mathrm{C}_{\mathrm{AgNO} 3}$ representa a concentração da solução de $\mathrm{AgNO}_{3}, \mathrm{~V}_{\mathrm{AgNO} 3}$ o volume de $\mathrm{AgNO}_{3}$ gasto na titulação da solução de cloridrato de metformina na presença do indicador cromato de potássio, $\mathrm{V}_{\text {met }} \mathrm{o}$ volume de solução de metformina titulado e $\mathrm{C}_{\text {met }}$ a concentração da solução de metformina. $\mathrm{O}$ volume de nitrato de prata teórico foi calculado usando essa fórmula.

Antes do início da titulação da solução aquosa do cloridrato de metformina com nitrato de prata, a concentração de cloreto na solução é elevada. Como o cloreto de prata $(\mathrm{AgCl})\left(\mathrm{s}=1,1 \times 10^{-5}\right.$ mol L-1) é menos solúvel que o cromato de prata $\left(\mathrm{Ag}_{2} \mathrm{CrO}_{4}\right)\left(\mathrm{s}=1,2 \times 10^{-4} \mathrm{~mol} \mathrm{~L}^{-1}\right)$ (HARRIS, 2005), o sal que precipita primeiro é o $\mathrm{AgCl}$, seguido então do $\mathrm{Ag}_{2} \mathrm{CrO}_{4}$.

Iniciada a titulação, a concentração do íon cloreto diminui em consequência da reação de precipitação e da diluição da solução (OHLWEILER, 1980). Até o ponto de equivalência, os íons $\mathrm{Cl}^{-}$da molécula de metformina reagem com os íons $\mathrm{Ag}^{+}$(aq) do titulante formando o precipitado de $\mathrm{AgCl}$ (Eq. 4). Após o ponto de equivalência, uma pequena adição do titulante é suficiente para precipitar o cromato de prata de cor vermelha indicando o ponto final da titulação (Eq. 5).

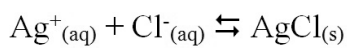

$\mathrm{Ks}(\mathrm{AgCl})=1,2 \times 10^{-10}=\left[\mathrm{Ag}^{+}\right]\left[\mathrm{Cl}^{-}\right]$

(Eq. 4)

$2 \mathrm{Ag}^{+}+\mathrm{CrO}_{4}^{2-} \leftrightarrows \mathrm{Ag}_{2} \mathrm{CrO}_{4(\mathrm{~s})}$

$\mathrm{Ks}\left(\mathrm{Ag}_{2} \mathrm{CrO}_{4}\right)=1,7 \times 10^{-12}=\left[\mathrm{Ag}^{+}\right]^{2}\left[\mathrm{CrO}_{4}^{2-}\right]$

(Eq. 5)

Realizaram-se três titulações de cada uma das amostras de medicamentos em concentração aproximada de $0,10 \mathrm{~mol} \mathrm{~L}^{-1}$, utilizando $\mathrm{AgNO}_{3}$ $0,10 \mathrm{~mol} \mathrm{~L}^{-1}$ na presença do indicador $\mathrm{K}_{2} \mathrm{CrO}_{4}$. A Tabela 1 mostra os resultados obtidos na titulação de duas amostras de medicamentos contendo cloridrato de metformina.
Tabela 1 - Teores de cloridrato de metformina obtidos empregando-se o método espectrofotométrico de referência e o Método de Mohr

\begin{tabular}{cccccc}
\hline \multicolumn{4}{c}{ MET (mg/comprimido) } & \multicolumn{2}{c}{} \\
Amostras/Rotulado & Referência $^{\mathrm{a}}$ & Mohr $^{\mathrm{a}}$ & $\begin{array}{c}\mathrm{ER}_{1}{ }^{\mathrm{b}} \\
\%\end{array}$ & $\begin{array}{c}\mathrm{ER}_{2}{ }^{\mathrm{c}} \\
\%\end{array}$ \\
A & 500 & $497 \pm 7$ & $495 \pm 3$ & $-1,0$ & $-0,4$ \\
B & 800 & $851 \pm 5$ & $865 \pm 5$ & 1,8 & 1,6 \\
\hline
\end{tabular}

${ }^{\mathrm{a}} \mathrm{n}=3 ;{ }^{\mathrm{b}} \mathrm{RE}_{1}=100 \times$ (Método de Mohr - valor rotulado) valor rotulado; ${ }^{\mathrm{C}} \mathrm{RE}_{2}=100 \times($ Método de Mohr - método de referência) / método de referência.

Fonte: Dados do autor.

O teste-t pareado foi aplicado aos resultados obtidos empregando ambos os métodos, resultando em um valor de $\mathrm{t}$ calculado de 0,416 , menor que o valor de $\mathrm{t}$ tabelado (12,706), indicando que o procedimento proposto possui boa exatidão, uma vez que não foi observada diferença significativa entre os resultados obtidos, a um nível de confiança de $95 \%$.

A concentração de cloridrato de metformina foi calculada empregando-se a Eq. 3 e, como etapa seguinte, para obter o resultado final da análise em mg de cloridrato de metformina /comprimido empregou-se a seguinte equação (Eq. 6):

teor de met $=\left(\frac{\mathrm{C}_{\text {met }} \times \mathrm{M}_{\text {met }} \times \mathrm{V}_{\mathrm{a}}}{1000}\right)\left(\frac{\mathrm{m}_{1}}{\mathrm{~m}_{2}}\right)$

(Eq. 6)

sendo $\mathrm{M}_{\text {met }}$ igual a $165,63 \mathrm{~g} \mathrm{~mol}^{-1}$ (massa molar do cloridrato de metformina), $\mathrm{V}_{\mathrm{a}}$ o volume em que a quantidade de comprimido pesada $\left(\mathrm{m}_{2}\right)$ foi dissolvida e m1 a média das massas obtidas na pesagem dos 5 comprimidos.

O volume gasto para obter a coloração vermelha na titulação do branco, que correspondeu a 0,02 $\mathrm{mL}$, não foi levado em conta nos cálculos, por ser praticamente desprezível. O erro da titulação somente torna-se apreciável para soluções diluídas, na ordem de $0,01 \mathrm{~mol} \mathrm{~L}^{-1}$ (JEFFERY et al., 1989).

$\mathrm{O}$ efeito de interferentes em potencial na determinação do cloridrato de metformina em 
medicamentos foi avaliado para excipientes normalmente presentes em amostras comerciais (povidona, estearato de magnésio, celulose, polietilenoglicol, dióxido de titânio, parafina e sorbitol). Não foi observada interferência nos valores de volume de equivalência encontrados para esses interferentes estudados. A maioria destes excipientes possui baixa solubilidade e foram separados por filtração da solução da amostra que foi titulada.

\section{Conclusões}

A contextualização do método de Mohr na determinação do cloridrato de metformina em medicamentos, proposto neste experimento, é um método simples e de baixo custo, totalmente viável para ser ministrado como prática experimental em uma disciplina de Química Analítica para estudantes de graduação de química ou de farmácia, principalmente em laboratórios com poucos recursos materiais.

Esse contexto promove um elo entre o dia-adia dos estudantes e o ensino da teoria e prática de química ou de farmácia, uma dimensão dinâmica do processo educativo. Ademais, fornece ao professor um embasamento teórico necessário antes do trabalho em laboratório, que facilita o aproveitamento do experimento pelos estudantes e consequentemente, uma assimilação significativa do conteúdo abordado.

\section{Agradecimentos}

Os autores agradecem à Coordenação de Aperfeiçoamento de Pessoal de Nível Superior (CAPES), ao Conselho Nacional de Desenvolvimento Científico e Tecnológico (CNPq) e à Fundação de Amparo à Pesquisa do Estado de São Paulo (FAPESP) pelo suporte financeiro e pelas bolsas concedidas.

\section{Referências}

BACCAN, N.; ANDRADE, J. C.; GODINHO, O. E. S.; BARONE, J. S. Química Analitica Quantitativa Elementar. 3. ed. São Paulo: Edgard Blücher, 2001.

BRASIL. Ministério da Educação. Lei no 9.394 de 20 de Dezembro de 1996. Estabelece as diretrizes e bases da educação nacional. 1996.

METFORMIN HYDROCHLORIDE. In: British Pharmacopoeia. London: HMSO, 1998. p. 1802.

FATIBELLO-FILHO, O.; WOLF, L. D.; ASSUMPÇÃO, M. D. M. T.; LEITE, O. D. Experimento simples e rápido ilustrando a hidrólise de sais. Química Nova na Escola, São Paulo, v. 24, p. 30, 2006.

HARRIS, D. C. Análise química quantitativa. 6. ed. Rio de Janeiro: LTC, 2005.

JEFFERY, G. H.; BASSET, J.; MENDHAM, J.; DENNEY, R. C. Vogel's Textbook of quantitative chemical analysis. 5. ed., England: Longman Scientific \& Technical, 1989.

KOLTHOFF, I. M.; SANDELL, E. B.; MEEHN, E. J.; BRUCKENSTEIN, S. Quantitative chemical analysis, 4. ed. Londres: The Mcmillan Company, 1969.

LUPETTI, K. O.; CARVALHO, L. C.; MOURA, A. F.; FATIBELLO-FILHO, O. Análise de imagem em química analítica: empregando metodologias simples e didáticas para entender e prevenir o escurecimento de tecidos vegetais. Química Nova, São Paulo, v. 28, p. 548-554, 2005 .

OHLWEILER, O. A. Química analitica quantitativa. 2. ed. Rio de Janeiro: Livros Técnicos e Científicos, 1980.

RAMOS, L. A.; LUPETTI, K. O.; CAVALHEIRO, E. T. G.; FATIBELLO-FILHO, O. Utilização do extrato bruto de frutos de Solanum nigrum L. no ensino de Química. Eclética Química, v. 25, p. 229-240, 2000.

SARTORI, E. R.; BATISTA, E. F.; FATIBELLO-FILHO, O. Escurecimento e limpeza de objetos de prata - um experimento simples e de fácil execução envolvendo reações de oxidação-redução. Química Nova na Escola, v. 30 , p. $61-65,2008$.

SARTORI, E. R.; BATISTA, E. F.; SANTOS, V. B.; FATIBELLO-FILHO, O. Construção e aplicação de um destilador como alternativa simples e criativa para a compreensão dos fenômenos ocorridos no processo de destilação. Química Nova na Escola, v. 31, p. 55-57, 2009 .

SILVA, P. Farmacologia. 6. ed. Guanabara Koogan: Rio de Janeiro, 2002. 
SUAREZ, W. T.; FERREIRA, L. H.; FATIBELLOFILHO, O. Padronização de soluções ácida e básica empregando reagentes do cotidiano. Química Nova na Escola, v. 25, p. 36-38, 2007.

VIEIRA, H. J.; FIGUEIREDO-FILHO, L. C. S.; FATIBELLO-FILHO, O. Um experimento simples e de baixo custo para compreender a osmose. Química Nova na Escola, v. 26, p. 40-43, 2007. 
